\title{
Analyse du discours médical sur Twitter®. Étude d'un corpus de tweets émis par des médecins généralistes entre juin 2012 et mars 2017 et contenant le hashtag \#DocTocToc
}

\author{
A. Salles ${ }^{a}$, J. Dufour ${ }^{a}$, P. Hassanaly ${ }^{a}$, P. Michel ${ }^{b}$, C. Cabot ${ }^{c, e}$, J.Grosjean ${ }^{c, d}$ \\ a Aix Marseille Université, UMR 1252 SESSTIM, Marseille, France \\ b Aix Marseille Université, Aix Marseille School of Economics (AMSE), Marseille, France \\ c CHU de Rouen, Département d'informatique et d'informations médicales du CHU Hôpitaux de \\ Rouen, Rouen, France \\ d Inserm, Département d'informatique biomédicale, LIMICS U1142, Paris, France \\ e Normandie Université TIBS-LITIS EA 4108, Saint-Etienne-du-Rouvray, France
}

\section{Introduction}

Les technologies de l'information et de la communication ont permis la naissance du web 2.0, caractérisé par la mise en place et l'utilisation de nouveaux outils collaboratifs de communication tels que les blogs, les wikis, les fils RSS et les réseaux sociaux. En s'appropriant ces outils, une médecine participative basée sur le partage d'informations et d'expériences entre professionnels, patients et tout acteur de la santé s'est développée. Depuis juin 2012, une communauté médicale échange sur Twitter avec le hashtag \#DocTocToc et contribue à la naissance de la e-santé sur ce réseau social. L'objectif de cette étude est d'analyser les principales thématiques des demandes effectuées via le hashtag \#DocTocToc par les médecins généralistes entre juin 2012 et mars 2017.

\section{Méthodes}

Une collecte de données par une méthode de «web scraping » a permis de constituer un corpus de tweets dont les auteurs ont été identifiés manuellement afin de procéder à un échantillonnage, de façon à ne conserver que les tweets émis par les médecins généralistes. Une étape de prétraitement a permis de transformer les formes potentiellement non reconnues par les logiciels de traitement du langage naturel. Le corpus a été appréhendé à l'aide de deux approches : une approche lexicale via le logiciel Iramuteq ${ }^{\circledR}$ et une indexation terminologique par l'extracteur de concepts multi-terminologiques (ECMT) du Catalogue et index des sites médicaux francophones (CISMeF).

\section{Résultats}

Sur les 12716 tweets recueillis, 7366 étaient rédigés par des médecins généralistes et ont été analysés. L'approche lexicale détermine deux grands mondes lexicaux représentés sous forme de dendrogramme, l'un en lien avec les demandes médico administratives relatives à la gestion du cabinet et à la prise en charge sociale du patient, l'autre en lien avec les demandes d'ordre purement médicales. La méthode d'indexation terminologique met en évidence les spécialités médicales pourvoyeuses de demandes de télé-expertise : gynécologie, neurologie, infectiologie, pédiatrie, cardiologie, dermatologie ; et permet de les croiser avec l'objectif de la demande : diagnostic, thérapeutique. 


\section{Conclusion}

Sur Twitter®, le hashtag \#DocTocToc est utilisé par les médecins généralistes comme un espace de partage informel d'informations en matière de santé mais aussi de gestion de problèmes administratifs et sociaux. Le DocsTocToc se présente comme un groupe d'échange de pratique à grande échelle ou le médecin compte sur l'avis de ses pairs.(Fig. 1)

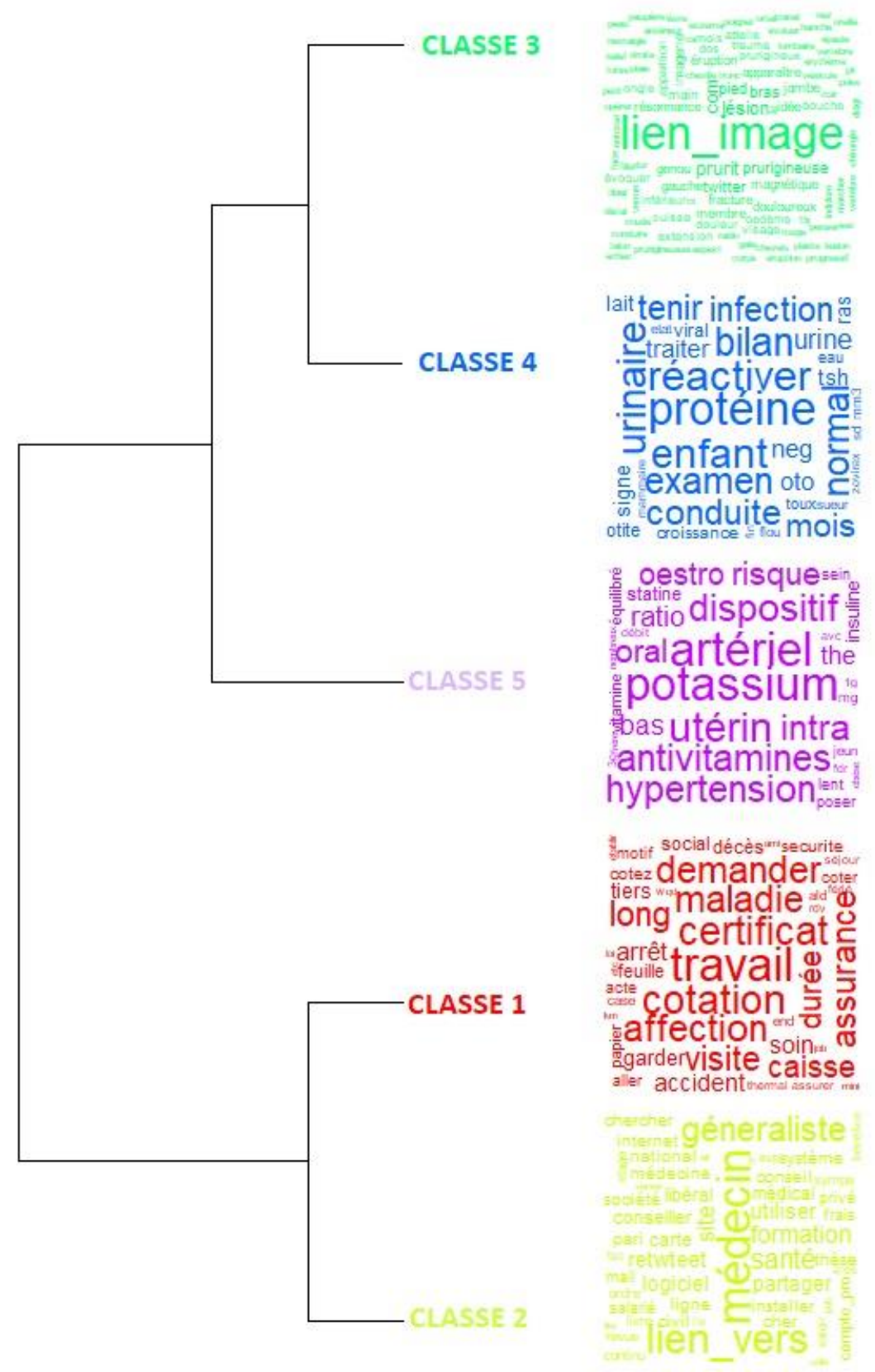

\section{Mots clés}

e-santé, Twitter, communication, big data, text mining 